\title{
Gain-Clamped Quantum Dot Semiconductor Optical Amplifiers
}

\author{
Omar Qasaimeh
}

\begin{abstract}
The linear optical gain of gain-clamped quantum dot semiconductor optical amplifiers (GCSOAs) has been investigated and studied for different energy detuning, doping concentration and different device length. Our analysis shows that large linear optical gain can be obtained when the laser emits $90 \mathrm{meV}$ above the ground state and the amplifier operates at the ground state which results in $81 \mathrm{meV}$ separation between the laser and amplifier energy. We find that larger detuning between the laser and amplifier energy result in gain saturation. Also we find that for positive $\hbar \omega_{L}-\hbar \omega_{A}$ doping the dots by P-type concentration enhances the linear optical gain while doping the dots by $\mathrm{N}$-type concentration reduces the linear optical gain. While for negative $\hbar \omega_{L}-\hbar \omega_{A}$ doping the dots by $\mathrm{N}$-type concentration enhances the linear optical gain while doping the dots by P-type concentration reduces the linear optical gain.
\end{abstract}

Index Terms-Quantum dot, gain-clamped, linear, semiconductor optical amplifier.

\section{INTRODUCTION}

Linear gain-clamped semiconductor optical amplifiers (GCSOAs) are essential elements in modern optical networks since conventional multichannel semiconductor optical amplifiers are characterized by large crosstalk between channels and significant intermodulation distortion due to the dependence of the gain on the device optical power. In gain-clamped semiconductor optical amplifier, the gain is clamped by several methods such as using distributed feedback, distributed Bragg reflector lasers and a vertical cavity laser [1]-[7].

Today, the fabrication and growth of quantum-confined heterostructures have created the opportunity to use quantum dots in the active layers of lasers and semiconductor optical amplifiers. This is attractive since quantum dots are capable of improving the electrical and optical properties of these devices [8]-[14]. The gain spectrum of arrays of quantum dots, which is fed through the wetting layer via capture/escape mechanisms, is formed from various dot groups, which makes the gain spectrum wide. The wide gain spectrum of quantum dot heterostructures can be exploited to realize broadband gain-clamped optical amplifiers. Another unique property of quantum dot ensembles is the inherently fast carrier capture and escape mechanisms that are very interesting in ultrafast applications. This work describes a detailed theoretical analysis of gain-clamped quantum dot semiconductor optical

Manuscript received December 9, 2013; revised March 18, 2014.

O. Qasaimeh is with the Electrical Engineering Department, Jordan University of Science and Technology, Irbid 22110 Jordan (e-mail: qasaimeh@just.edu.jo). amplifier for different energy detuning, doping concentration and different device length.

\section{THEORETICAL MODEL}

The investigated device is an edge emitting laser structure having arrays of self-organized quantum dots in the active layer. The structure acts as a single wavelength laser emitting at $\hbar \omega_{L}$ with no input where the lasing energy is determined by a two DBR mirrors at the edges of the structure. The laser is biased above threshold. One facet of the waveguide acts as the input of the amplifier and the other facet acts as the output of the amplifier. When a signal of photon energy $\hbar \omega_{A}$ is applied at the input of the amplifier, the laser will stop lasing and the input signal will be amplified linearly since the gain is clipped at threshold. Large detuning between $\hbar \omega_{L}$ and $\hbar \omega_{A}$ is required to enable filtering the amplified signal from the laser signal.

The photon rate equation that describes lasing in GCSOA is

$$
\frac{1}{v_{g}} \frac{\partial S^{L}}{\partial t}=\sum_{i=0}^{M_{n}} \sum_{j=0}^{M_{p}} a_{i j}^{L}\left(f_{i}^{n}+f_{j}^{p}-1\right) S^{L}-\alpha^{L} S^{L}
$$

And the photon rate equation for the amplified signal is

$$
\frac{\partial S^{A}}{\partial z}=\sum_{i=0}^{M_{n}} \sum_{j=0}^{M_{p}} a_{i j}^{A}\left(f_{i}^{n}+f_{j}^{p}-1\right) S^{A}-\alpha^{A} S^{A}
$$

where $t$ is time, the superscript $L$ stands for the laser and the superscript $A$ stands for the amplifier. $f_{i}^{n}$ and $f_{i}^{p}$ are the occupation probability for the electrons and holes in the i-th state where $i=0$ represents the ground state. $M_{n}$ and $M_{p}$ are the number of electron and hole states respectively. $S^{k}$ is the photon density where $k=L$ or $k=A, z$ is the distance, $\alpha^{k}$ is the effective loss coefficient. $a_{i j}^{k}$ is the material gain coefficient of the active layer which is a function of inhomogeneous line broadening [13]. where

$$
a_{i j}^{k}=g_{i j}^{\max } \frac{\hbar \omega_{i}^{\max }}{\hbar \omega^{k}} \operatorname{Exp}\left(\frac{-\left(\hbar \omega^{k}-\hbar \omega_{i}^{\max }\right)^{2}}{2 \sigma_{i}^{2}}\right)
$$

where $\sigma_{i}$ is the inhomogeneous line broadening, $g_{i j}^{\max }$ is the gain coefficient for the $\mathrm{i}$-th transition, $\hbar \omega^{k}$ is the photon 
energy of the laser or amplifier and $\hbar \omega_{i}^{\max }$ is the energy corresponding to the gain peak of the i-th transition.

For InAs quantum dots, the heterostructure has 3 energy levels in the conduction band and 8 energy levels in the valence band. The rate equation for electrons in the $\mathrm{i}$-th energy state is given by [13]

$$
\frac{\partial f_{i}^{n}}{\partial t}=\left(R_{i+1, i}^{n c}-R_{i, i+1}^{n e}\right)-\left(R_{i, i-1}^{n c}-R_{i-1, i}^{n e}\right)-\frac{f_{i}^{n} f_{i}^{p}}{\tau_{i R}}-R_{s t}
$$

where $R_{i+1, i}^{n c}$ is the electron capture rate from the (i+1)th state to the i-th state and $R_{i, i+1}^{n e}$ is the electron emission rate from the $\mathrm{i}$-th state to the $(\mathrm{i}+1)$ th state. $\tau_{i R}$ is the spontaneous radiative lifetime in $\mathrm{i}$-th state. In (4) the stimulation emission rate is given as

$$
R^{s t}=\sum_{k} \frac{v_{g} a_{i}^{k}\left(f_{i}^{n}+f_{i}^{p}-1\right) S^{k}}{N_{Q}}
$$

The capture and emission rates are respectively given as [13]

$$
\begin{aligned}
& R_{i+1, i}^{n c}=\frac{\left(1-f_{i}^{n}\right) f_{i+1}^{n}}{\tau_{i+1, i}^{n}}\left(b_{i+1, i}^{n}+c_{i+1, i}^{n p} f_{w}^{p}+c_{i+1, i}^{n n} f_{w}^{n}\right) \\
& R_{i, i+1}^{n e}=\frac{f_{i}^{n}\left(1-f_{i+1}^{n}\right)}{\tau_{i, i+1}^{n}}\left(b_{i+1, i}^{n}+c_{i+1, i}^{n p} f_{w}^{p}+c_{i+1, i}^{n n} f_{w}^{n}\right)
\end{aligned}
$$

where $f_{w}^{p}$ and $f_{w}^{n}$ are respectively the occupation probability for the holes and electrons in the wetting layer, $\tau_{i+1, i}^{n}$ is the electron capture time from the $\mathrm{i}+1$ state to the $\mathrm{i}$-th state and $\tau_{i, i+1}^{n}$ is the electron escape time from the $\mathrm{i}$-th state to the $i+1$ state. $b_{i+1, i}^{n}$ is the phonon-assisted coefficient, $c_{i+1, i}^{n n}$ is the electron-electron Auger-assisted coefficient and $c_{i+1, i}^{n p}$ is the electron-hole Auger-assisted coefficient.

The rate equation for the wetting layer state is

$$
\frac{\partial f_{w}^{n}}{\partial t}=\frac{J}{\tau_{w R}}-\left(\frac{\left(1-f_{M_{n}}^{n}\right) f_{w}^{n}}{\tau_{w, M_{n}}^{n}}-\frac{f_{M_{n}}^{n}\left(1-f_{w}^{n}\right)}{\tau_{M_{n}, w}^{n}}\right)-\frac{f_{w}^{n}}{\tau_{w R}}
$$

where $f_{w}^{n}$ is the occupation probability for the electrons in the wetting layer, $I$ is the applied current, $q$ is the charge of electron, $V_{a}$ is the volume and $N_{W L}$ is the carrier density for the wetting layer. $\tau_{w, M_{n}}^{n}$ is the electron capture time from the wetting layer to the $M_{n}$ state and $\tau_{M_{n}, w}^{n}$ is the electron escape time from the $M_{n}$ state to the wetting layer, and $\tau_{w R}$ is the spontaneous radiative lifetime in wetting layer.

Under quasi-Fermi equilibrium condition, the capture and escape lifetime are related to each other via $\tau_{i, i+1}^{n, p} / \tau_{i+1, i}^{n, p}=\operatorname{Exp}\left(\Delta_{i+1, i}^{n, p} / K T\right) \quad$ where $K$ is Boltzmann constant, $T$ is the absolute temperature and $\Delta_{i+1, i}^{n, p}$ is the energy separation between the $i$ and the $i+1$ states.

Similar rate equations are modeled for the hole states. The relation between the electron and the hole concentration is determined by the charge neutrality equation which is given by

$$
N_{A}+\sum_{i=0}^{M_{n}} N_{i} f_{i}^{n}+n_{w}=N_{D}+\sum_{j=0}^{M_{p}} N_{j} f_{j}^{p}+p_{w}
$$

where $N_{j}$ is the volume density of the $j$-th state, $N_{A}$ and $N_{D}$ are the acceptor and donor concentrations respectively, and $n_{w}$ and $p_{w}$ are the electron and the hole concentration of the wetting layer.

\section{RESULTS AND DISCUSSION}

The InAs QD heterostructure has the following parameters: $\tau_{R}=1 \mathrm{~ns}, N_{q}=2.5 \times 10^{17} \mathrm{~cm}^{3}, \mathrm{v}_{\mathrm{g}}=8.45 \times 10^{9} \mathrm{~cm} / \mathrm{s}, \tau_{10}^{n}=8 \mathrm{ps}$, $\tau_{i+1, i}^{n}=2 p s, \tau_{k+1, k}^{p}=1 p s$. The separations of the electron and hole energy states are $60 \mathrm{meV}$ and $10 \mathrm{meV}$ respectively. The gain coefficients for the active layer are $g_{00}^{\max }=14 \mathrm{~cm}^{-1}, g_{11}^{\max }=20 \mathrm{~cm}^{-1}$, and $g_{22}^{\max }=10 \mathrm{~cm}^{-1}$. The inhomogeneous line broadening is $30 \mathrm{meV}$. The material loss is $7 \mathrm{~cm}^{-1}$.

With no input the laser is lasing and the gain is clamped by threshold condition. When a signal is applied at the input of the SOA, the signal will be linearly amplified and the laser output will be reduced. The photon energy of the laser $\hbar \omega_{L}$ is selected to be far away from the amplifier photon energy $\hbar \omega_{A}$ to maximize the optical gain and to have large linear gain. For simplicity, we choose $S^{A}(z=0)=0.1 S_{R}$ where $S_{R}=N_{Q}\left(v_{g} a_{00} \tau_{0 R}\right)^{-1}$ which is fairly enough to study the linear gain of the amplifier. The linear optical gain of GCSOA as a function of the amplifier photon energy is shown in Fig. 1 for different value of laser energy $\left(\hbar \omega_{L}\right)$. For simplicity, we express the photon energy in term of the ground state (GS) energy, i.e, $\hbar \omega_{L}=0 \mathrm{meV}$ means that the laser is lasing from the ground state. Similar thing is used for $\hbar \omega_{A}$. As evident from Fig. 1, the peaks of the optical gain occur at $\hbar \omega_{A}=9 \mathrm{meV}$ and $65 \mathrm{meV}$ which are determined by the energies of the ground state and excited state, respectively. As $\hbar \omega_{L}$ increases the magnitude of the two peaks increases. Large separation between $\hbar \omega_{L}$ and $\hbar \omega_{A}$ is obtained when $\hbar \omega_{L}$ is at $90 \mathrm{meV}$ and $\hbar \omega_{A}$ is at the $9 \mathrm{meV}$ which provide a linear optical gain as large as $7.5 \mathrm{~dB}$. One advantage of this device is the large separation between the laser emission wavelength and the amplifier wavelength, which can be as high as $81 \mathrm{meV}$ which facilitates easy filtering of the laser power from the output signal. Increasing $\hbar \omega_{L}$ above 
$95 \mathrm{meV}$ result in quenching the lasing mechanism which results in gain saturation at the gain peak. Since we are looking for linear amplifier the value of $\hbar \omega_{L}$ should not exceed $95 \mathrm{meV}$. The effect of increasing the length of the gain region on the optical gain is calculated as shown in Fig. 2 for $\hbar \omega_{L}=90 \mathrm{meV}$. As evident, increasing the gain region length improves the optical gain.

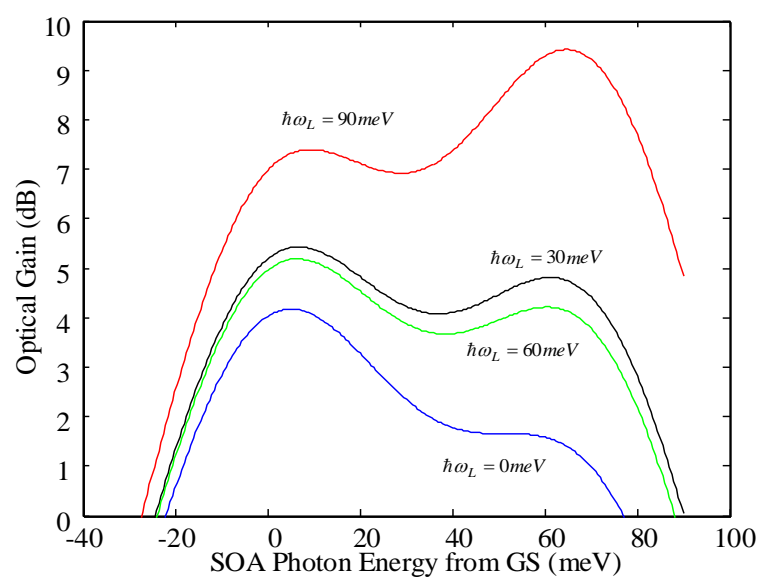

Fig. 1. Optical gain as a function of SOA photon energy.

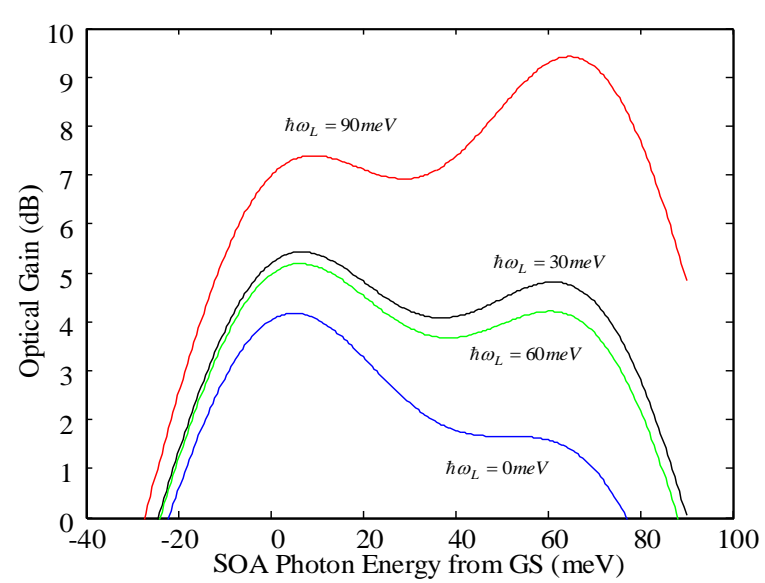

Fig. 2. Optical gain for different gain length for $\hbar \omega_{L}=90 \mathrm{meV}$.

In quantum dots the hole effective mass is large and band mixing is strong. Due to that the energy band of quantum dot active layers lave large numbers of hole states in the valence band and few number of electron states in the conduction band. The hole states have small energy separation $(\sim 10 \mathrm{meV})$ [13] and the electron state have larger energy separation $(\sim 60 \mathrm{meV})$. Since the energy separation between the hole states is small, thermal effects are significant. Due to that the injected holes are thermally broadened which decreases the QD ground state gain and increases the temperature sensitivity of the device. It has been experimentally demonstrated that doping the dots with p-type can efficiently provide excess holes which improves the gain properties and increases the room-temperature modulation speed of QD lasers [13]. Moreover, significant improvement of the linewidth enhancement factor in QD lasers has been achieved when the dots are doped with p-type concentration [13].

The effect of doping the active layer on the optical gain of quantum dot GCSOA is studied. The doping concentration of the quantum dot layer is varied and the optical gain of the amplifier is calculated. The optical gain for different doping concentrations is shown in Fig. 3 for laser emission wavelength fixed at $\hbar \omega_{L}=90 \mathrm{meV}$. As evident, for positive $\hbar \omega_{L}-\hbar \omega_{A}$ detuning, doping the dots by P-type concentration enhances the linear optical gain while doping the dots by $\mathrm{N}$-type concentration reduces the optical gain. This finding is different from the finding in Ref. [14] for quantum dash GCSOA which is due to the difference in the band structure of quantum dot. In quantum dash structure the band structure has large and closely spaced conduction band states. Fig. 3 shows that doping the dots by $N_{A}=2 \times 10^{9} \mathrm{~cm}^{-2}$ increases the linear gain by $\sim 3 \mathrm{~dB}$ at $\hbar \omega_{A}=9 \mathrm{meV}$ (with linear optical gain of about $10 \mathrm{~dB}$ ). When the p-type concentration is increased to $N_{A}=4 \times 10^{9} \mathrm{~cm}^{-2}$ the optical gain is increased and around $\hbar \omega_{A}=9 \mathrm{meV}$ the gain become non-linear as indicated by circles in Fig. 3. In this region the amplifier operates as a saturated amplifier.

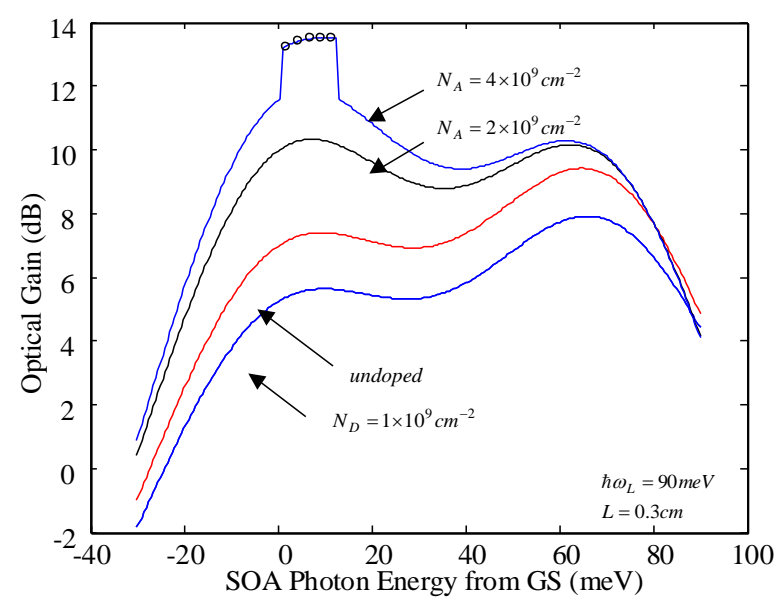

Fig. 3. Optical gain for different doping for positive detuning.

The analysis is repeated for negative $\hbar \omega_{L}-\hbar \omega_{A}$ detuning. The optical gain is shown in Fig. 4 for $\hbar \omega_{L}=0$. As evident, for negative $\hbar \omega_{L}-\hbar \omega_{A}$ detuning, doping the dots by P-type concentration reduces the linear optical gain while doping the dots by N-type concentration enhances the optical gain. Fig. 4 shows that doping the dots by $N_{D}=1.5 \times 10^{9} \mathrm{~cm}^{-2}$ increases the linear gain by $\sim 8.5 \mathrm{~dB}$ at $\hbar \omega_{A}=67 \mathrm{meV}$ (with linear optical gain of about $9 \mathrm{~dB}$ which is about the same linear optical gain in positive detuning).

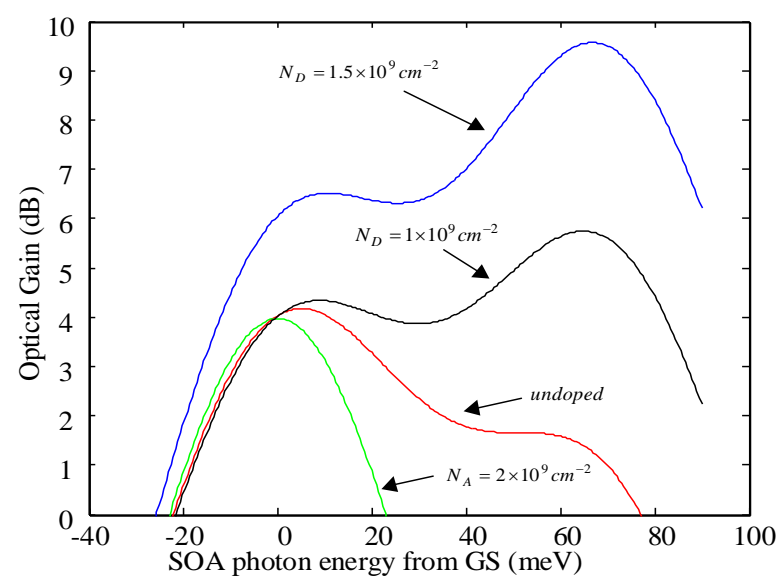

Fig. 4. Optical gain for different doping for negative detuning. 


\section{CONCLUSION}

The gain characteristics of undoped and doped gain-clamped quantum dot semiconductor optical amplifiers have been studied. We find that large linear optical gain can be obtained when the laser emits at $90 \mathrm{meV}$ above the ground state and when the photon energy of the amplifier is at the ground state energy. Large separation between the laser and amplifier energy (as large as $81 \mathrm{meV}$ ) is obtained. We find that detuning between the laser and amplifier energy (larger than $81 \mathrm{meV}$ ) result in non-linear gain saturation. Our analysis also reveals that doping the dots by P-type concentration will enhance the linear optical gain while doping the dots by $\mathrm{N}$-type concentration will reduce the linear optical gain.

\section{REFERENCES}

[1] D. Wolfson, S. L. Danielsen, C. Joergensen, B. Mikkelsen, and K. E. Stubkjaer, "Detailed theoretical investigation of the input power dynamic range for gain-clamped semiconductor optical amplifier gates at $10 \mathrm{~Gb} / \mathrm{s}$," IEEE Photonics Tech. Lett., vol. 10, no. 9, pp. 1241-1243, 1998.

[2] C. Michie, A. E. Kelly, I. Armstrong, I. Andonovic, and C. Tombling, "An adjustable gain-clamped semiconductor optical amplifier (AGC-SOA)," IEEE J. Lightwave Technology, vol. 25, no. 6, pp. 1466-1473, 2007.

[3] G. X. Chen, W. Li, C. L. Xu, W. P. Huang, and S. S. Jian, "Time and spectral domain properties of distributed-feedback-type gain-clamped semiconductor optical amplifiers," IEEE Photonics Tech. Lett., vol. 18 no. 8, pp. 932-934, 2006

[4] J. Park, X. Li, and W. P. Huang, "Performance simulation and design optimization of gain-clamped semiconductor optical amplifiers based on distributed Bragg reflectors," IEEE J. Quantum Electron, vol. 39, no. 11, pp. 1415-1423, 2003.

[5] A. Bilenca and G. Eisenstein, "On the noise properties of linear and nonlinear quantum-dot semiconductor optical amplifiers: the impact of inhomogeneously broadened gain and fast carrier dynamics," IEEE $J$. Quantum Electron, vol. 40, no. 6, pp. 690-702, 2004.

[6] K. Vyrsokinos, G. Toptchiyski, and K. Petermann, "Comparison of gain clamped and conventional semiconductor optical amplifiers for fast all-optical switching," IEEE J. Lightwave Technology, vol. 20, no. 10, pp. 1839-1846, 2002.

[7] J. Park, X. Li, and W. P. Huang, "Gain clamping in semiconductor optical amplifiers with second-order index-coupled DFB grating," IEEE J. Quantum Electron, vol. 41, no. 2, pp. 366-375, 2005.
[8] L. Jiang and L. V. Asryan, "Excited-state-mediated capture of carriers into the ground state and the saturation of optical power in quantum-dot lasers," IEEE Photonics Tech. Lett., vol. 18, no. 24, pp 2611-2613, 2006.

[9] D. Deppe, H. Huang, and O. B. Shchekin, "Modulation characteristics of quantum-dot lasers: the influence of P-Type doping and the electronic density of states on obtaining high speed," IEEE J. Quantum Electron, vol. 38, no. 12, pp. 1587-1593, 2002.

[10] J. Kim and S. L. Chuang, "Theoretical and experimental study of optical gain, refractive index change, and linewidth enhancement factor of p-doped quantum-dot lasers", IEEE J. Quantum Electron, vol. 42, no. 9, pp. 942-952, 2006.

[11] H. C. Wong, G. B. Ren, and J. M. Rorison, "The constraints on quantum-Dot semiconductor optical amplifiers for multichannel amplification," IEEE Photonics Tech. Lett., vol. 18, no. 20, pp. 2075-2077, 2006.

[12] M. T. Todaro, A. Salhi, L. Fortunato, R. Cingolani, A. Passaseo, M. De Vittorio, P. Della Casa, F. Ghiglieno, and L. Bianco, "High-performance directly modulated 1.3- m undoped in as-In GaAs quantum-dot lasers," IEEE Photonics Tech. Lett., vol. 19, no. 4, pp 191-193, 2007.

[13] O. Qasaimeh, "Effect of doping on the optical characteristics of quantum dot semiconductor optical amplifiers," IEEE J. Lightwave Tech., vol. 27, no. 12, pp. 1978-1984, 2009.

[14] O. Qasaimeh, "Broadband gain-clamped linear quantum dash optical amplifiers," Optical and Quantum Electronics, vol. 45, no. 12, pp 1277-1286, 2013.

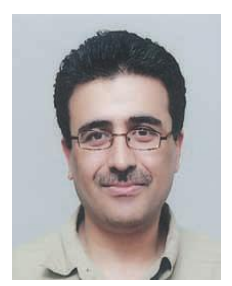

Omar Qasaimeh received the B.S. and M.S. degrees in electrical engineering from Jordan University of Science and Technology, Irbid, Jordan, in 1992 and 1994, respectively, and the Ph.D. degree in electrical engineering from the University of Michigan, Ann Arbor, in 2000. From 2000 to 2002, he was with Agere systems (now LSI), Breinigsville, PA, where he was engaged in research on the development of tunable distributed Bragg reflector lasers. He is currently a professor at Jordan University of Science and Technology. He is the author or coauthor of more than 90 papers published in technical journals and international conferences. His current research interests include design, fabrication, characterization, and development of high-efficiency, high-speed optoelectronic devices and integrated circuits using quantum dot active layers, including heterojunction phototransistors, vertical cavity surface-emitting lasers, microcavities, optical amplifiers and modulators, and tunable lasers. 